\title{
Catalan Traffic at the Beach
}

\author{
Heinrich Niederhausen \\ Department of Mathematical Sciences \\ Florida Atlantic University, Boca Raton, USA \\ Niederhausen@math.fau.edu
}

Submitted: March 17, 2002; Accepted: August 12, 2002.

MR Subject Classifications: 05A15, 05A19

\begin{abstract}
The ubiquitous Catalan numbers $C_{n}=\left(\begin{array}{c}2 n \\ n\end{array}\right) /(n+1)$ occur as $t(n,-n)$ in the following table showing the number of ways to reach the point $(n, m)$ on a rectangular grid under certain traffic restrictions, indicated by arrows.

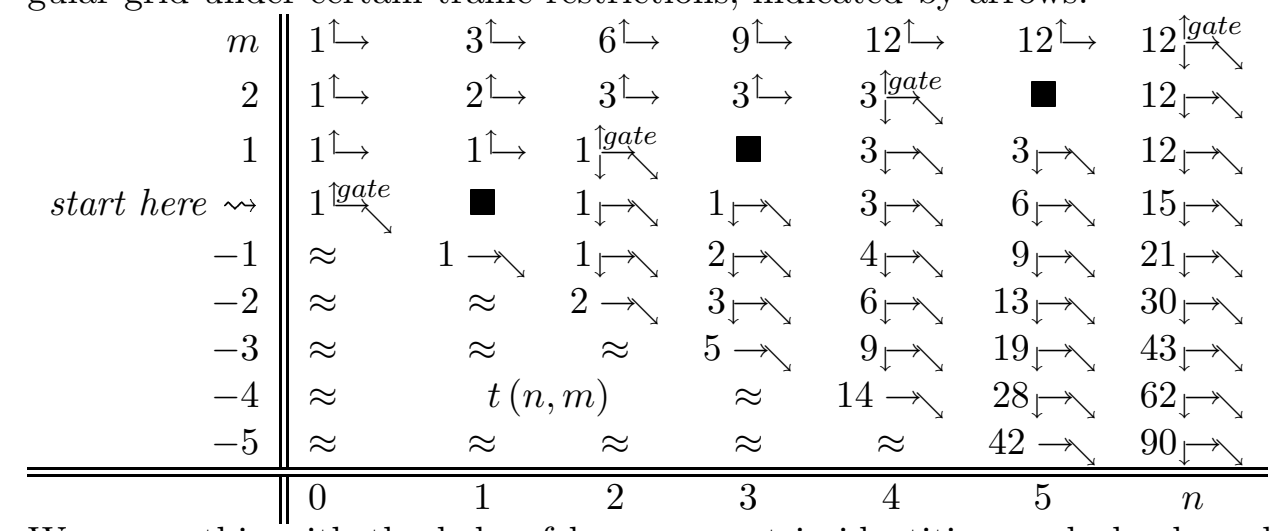

We prove this with the help of hypergeometric identities, and also by solving an equivalent lattice path problem. On the way we pick up several identities and discuss other known sequences of numbers occurring in the Catalan traffic scheme, like the Motzkin numbers in row $m=-1$, and the "Tri-Catalan numbers" $1,1,3,12,55, \ldots$ at the gates.
\end{abstract}

\section{Catalan Traffic}

There are 66 problems in Stanley's book Enumerative Combinatorics, Vol. II [10, Chpt. 6], about different combinatorial structures counted by the Catalan numbers $C_{i}=\frac{1}{i+1}\left(\begin{array}{c}2 i \\ i\end{array}\right)$. One example (Ballot problem, or Dyck paths) is the total count of lattice paths with step vectors East $(\rightarrow)$ and South $(\downarrow)$, starting at the origin, ending on the diagonal $y+x=0$, never going below that line. If we think of the lattice as the streets on a city map, the diagonal as the beach, we are talking about the number of shortest trips between two spots at the beach. 


\begin{tabular}{|c|c|c|c|c|c|c|}
\hline start here $\rightsquigarrow$ & $1^{\rightarrow}$ & $1 \vec{\downarrow}$ & $1_{\downarrow}$ & $1_{\downarrow} \vec{\imath}$ & $1_{\downarrow}$ & $1_{\downarrow}$ \\
\hline & $\approx$ & $1 \stackrel{\rightarrow}{\rightarrow}$ & $2 \stackrel{\downarrow}{\downarrow}$ & $3 \stackrel{\downarrow}{\downarrow}$ & $4_{\downarrow}^{\stackrel{\rightarrow}{\longrightarrow}}$ & $5 \stackrel{\downarrow}{\rightarrow}$ \\
\hline & $\approx$ & $\approx$ & $2^{\rightarrow}$ & $5 \stackrel{\downarrow}{\vec{\downarrow}}$ & $9 \stackrel{\downarrow}{\longrightarrow}$ & $14_{\downarrow}^{\hookrightarrow}$ \\
\hline & $\approx$ & $\approx$ & $\approx$ & $5^{\rightarrow}$ & $14 \vec{\jmath}$ & $28 \vec{\jmath}$ \\
\hline & $\approx$ & $\approx$ & $\approx$ & $\approx$ & $14^{\rightarrow}$ & $42_{\downarrow}^{\rightarrow}$ \\
\hline & $\approx$ & $\approx$ & $\approx$ & $\approx$ & $\approx$ & $42^{\rightarrow}$ \\
\hline
\end{tabular}

Table 1: The number of routes from the starting point to any other point on the beach are the Catalan numbers

We will prove that the following obstacle course, a carefully designed system of detours and right turns, again allows us to visit the diagonal in the same (Catalan-) number of ways as before, with the added benefit that we cannot get off the diagonal anymore.

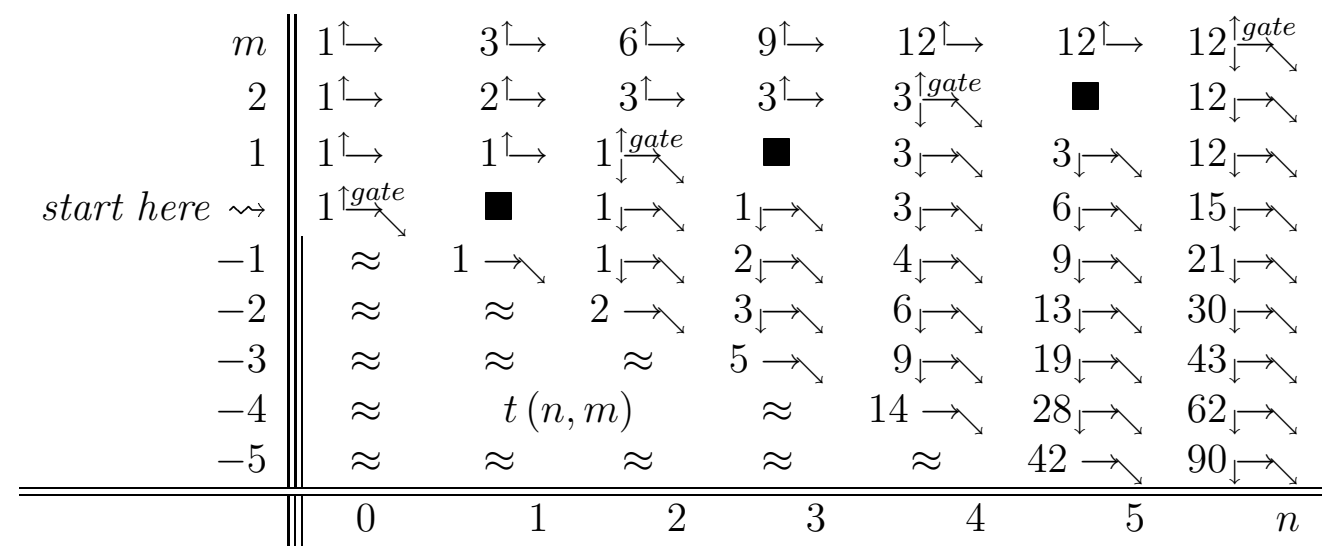

Table 2: Detours preserving Catalan traffic

The rules are as follows.

1. At lattice points strictly above the line $x=2 y$ go $\mathrm{N}$ or $\mathrm{E}(\uparrow$ and $\rightarrow)$.

2. At lattice points strictly below the line $x=2 y$ go $\mathrm{S}$ and $\mathrm{E}$, but enforce right turns when coming from the West. Note that such a right turn from $(i, j)$ to $(i+1, j-1)$ via $(i+1, j)$ is equivalent to a SE diagonal step $\searrow$. We could simply require that the step vectors are $\downarrow$ and $\searrow$.

3. Block out all traffic at the intersections $(2 m+1, m)$, except for right turns for paths from the West.

4. On the line, at the gates $(2 m, m)$, allow $\mathrm{N}, \mathrm{S}$, and right turn steps (because of the road block at $(2 m+1, m)$ these steps are equivalent to $\downarrow, \uparrow$, and $\searrow)$. The points on this line are the only gates from the upper traffic to the lower traffic.

Catalan traffic generates other known number sequences besides the Catalan numbers. As R. Sulanke pointed out to me, the sequence $t(n,-1), n \geq 1$, must be the sequence of 


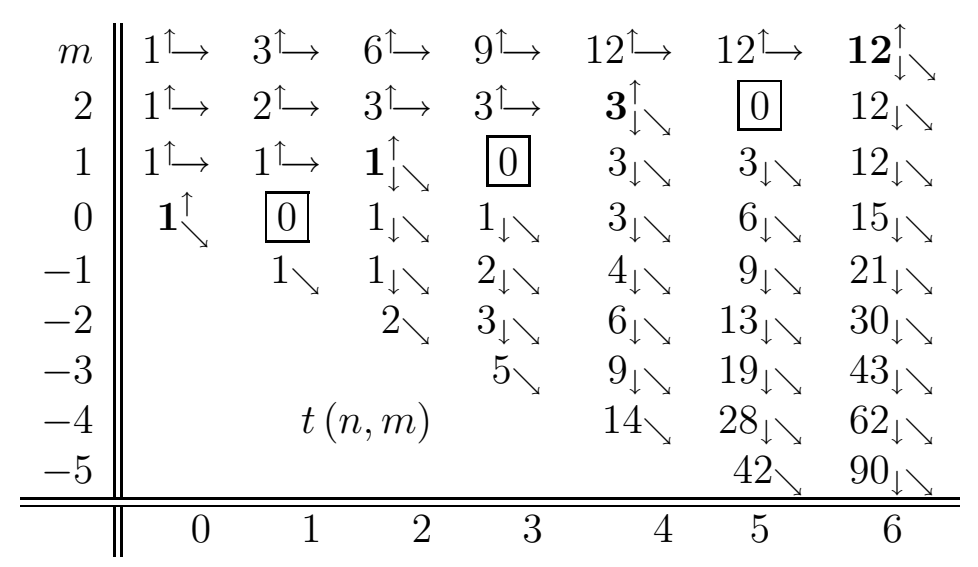

Table 3: Diagonal steps can replace the right turns (gates in bold)

Motzkin numbers if $t(n,-n)=C_{n}$. In addition, the numbers at the gates are also well known, $\left(\begin{array}{c}3 i \\ i\end{array}\right) /(2 i+1)$; they may be viewed as the "Tri-Catalan numbers" $C_{n}^{\prime \prime \prime}$ in the family $\left(\begin{array}{c}c n \\ n\end{array}\right) /((c-1) n+1)$ of Catalans. For references see the sequence A001764 in the On-Line Encyclopedia of Integer Sequences. They were the original motivation for devising the Catalan traffic when I was introduced to them in the form of a tennis ball sequence by Merlini, Sprugnoli, R., and Verri, M. C. [5]. Some of this additional material is compiled in Section 5. The three types of numbers that occur in the Catalan traffic scheme are summarized in the following table.

\begin{tabular}{l|l|l|l|}
$\begin{array}{l}\text { Number } a_{n} \\
\text { Catalan } C_{n}\end{array}$ & $\begin{array}{l}\text { Formula } \\
$\cline { 2 - 4 } \text {$\left.Tri-Catalan } C_{n}^{\prime \prime \prime} \\
n\end{array}\right) /(n+1)$ & $\frac{2}{1+\sqrt{1-4 t}}$ & $t(n,-n)$ \\
\cline { 2 - 4 } & $\left(\begin{array}{c}3 n \\
n\end{array}\right) /(2 n+1)$ & $\frac{2}{\sqrt{3 t}} \sin \left(\frac{1}{3} \arcsin (\sqrt{27 t} / 2)\right)$ & $t(2 n, n)$ \\
\cline { 2 - 4 } Motzkin $M_{n}$ & $\frac{1}{n+1} \sum_{k=0}^{n}\left(\begin{array}{c}k \\
n-k\end{array}\right)\left(\begin{array}{c}n+1 \\
k\end{array}\right)$ & $\frac{1}{2 t^{2}}(1-t-\sqrt{(1-3 t)(1+t)})$ & $t(n+1,-1)$ \\
\cline { 2 - 4 } & &
\end{tabular}

Table 4: Interesting numbers occuring in the Catalan traffic scheme

To prove that the numbers $t(n,-n)$ along the diagonal really are the Catalan numbers we will first reformulate the problem such that the same backwards difference recursion holds at every lattice point. Subsequently we give three proofs for the observation $t(n,-n)=C_{n}$. The first pair of proofs show that the following system of equations

$$
0=\sum_{i=0}^{2 n-1} x_{i}\left(\begin{array}{c}
3 n-2 \\
2 n-1-i
\end{array}\right) \text { and } 0=\sum_{i=0}^{2 n} x_{i}\left(\begin{array}{c}
3 n-1 \\
2 n-i
\end{array}\right)
$$

is (uniquely) solved by $x_{i}=(-1)^{i} C_{i-1}$ for $i>0$, and $x_{0}=1$. The first (and shortest) proof, Section 3.1, employs the help of Zeilberger's algorithm for hypergeometric functions to show that ${ }_{2} F_{1}\left[-m,-\frac{1}{2} ;\lceil m / 2\rceil ; 4\right]=3$ for positive integers $m$. The second proof, Section 3.2 , is also based on recursive evaluations of hypergeometric functions, offering 
more background material in the form of related identities (Corollary 3), like

$$
C_{n}^{\prime \prime \prime}=\frac{1}{2 n} \sum_{i=0}^{2 n-1}(-1)^{i} C_{i}\left(\begin{array}{c}
3 n \\
2 n-1-i
\end{array}\right), \quad C_{n}^{\prime \prime \prime}=\frac{1}{n+1} \sum_{i=0}^{2 n}(-1)^{i} C_{i}\left(\begin{array}{c}
3 n \\
2 n-i
\end{array}\right) .
$$

drawn from H.W. Gould's Improved evaluation of the finite hypergeometric series $F(-n, 1 / 2 ; j+1)[2]$ (Section 3.2). The third proof, in Section 4, is based on the known solution to the enumeration of lattice paths strictly above lines of slope 2. Combining the results of these different proofs establishes identities like

$$
\begin{aligned}
\sum_{i=0}^{m-n / 2}\left(\begin{array}{c}
2(m-i)-n \\
i
\end{array}\right) \frac{(-1)^{i}}{2(m-i)+1}\left(\begin{array}{c}
3(m-i) \\
2 m
\end{array}\right) & =\left(\begin{array}{c}
m+n \\
n
\end{array}\right)-\sum_{i=0}^{n-1}(-1)^{i} C_{i}\left(\begin{array}{c}
m+n \\
n-1-i
\end{array}\right) \\
\sum_{i=0}^{n-1}(-1)^{i} C_{i}\left(\begin{array}{c}
m+n \\
n-1-i
\end{array}\right) & =(-1)^{n} \sum_{i=0}^{(n-1) / 2} C_{i}^{\prime \prime \prime}\left(\begin{array}{c}
i-m-1 \\
n-1-2 i
\end{array}\right)
\end{aligned}
$$

for $m \geq(n-1) / 2$ (expansions (17) and (7)),

$$
\sum_{i=0}^{n / 2} C_{i}^{\prime \prime \prime}\left(\begin{array}{c}
n+i \\
3 i
\end{array}\right)=\frac{1}{n+1} \sum_{i=0}^{n / 2}\left(\begin{array}{c}
n+1 \\
2 i+1
\end{array}\right)\left(\begin{array}{c}
n+i \\
i
\end{array}\right)=C_{n}
$$

(expansion (18)), and

$$
\sin \left(\frac{1}{3} \arcsin \left(\frac{t}{2}\left(\frac{1-t}{3}\right)^{-3 / 2}\right)\right)=\frac{t \sqrt{3 /(1-t)}}{1+\sqrt{(1-4 t)}} .
$$

First, however, we transform the Catalan traffic at the beach into a problem about lattice paths with steps $\rightarrow, \uparrow$ strictly above the line $y=(x-1) / 2$ (Section 3.1), where the Catalan numbers occur again along the same diagonal, but with alternating signs. They will also occur when we count $\rightarrow, \uparrow$-paths strictly above the line $y=x / 2$. Such paths have been enumerated by Gessel [1] with his probabilistic approach. This and another approach valid for slopes of the form $1 / g$, where $g$ is a positive integer, are discussed in Section 5 .

\section{Untangling the Detours}

The numbers $t(n, m)$ in Table 3 can be recursively calculated from the given initial conditions. However, two simple changes will make the recursive calculations easier. In the first step we split the "gates" at $(2 m, m)$ into two cells, $(2 m, m)$ and $(2 m+1, m-1)$, duplicating the content, and shifting the whole matrix of numbers below the line $2 y=x$ one step SE. Call the new entries $t^{\prime}(n, m)$. We have $t^{\prime}(n, m)=t(n, m)$ if $n \leq 2 m$, $t^{\prime}(n, m)=t(n-1, m+1)$ if $n \geq 2 m+3$, and we fill the crack with zeroes, $t(2 m+1, m):=$ $t(2 m+2, m):=0$ for all $m \geq 0$. From the "new points" $(2 m, m),(2 m+1, m)$, and 


\begin{tabular}{|c|c|c|c|c|c|c|c|c|c|c|c|c|c|}
\hline \multicolumn{7}{|c|}{ Stretching the lattice (the crack in brackets) } & \multicolumn{7}{|c|}{ Changing signs } \\
\hline$m$ & $1 \uparrow$ & $3^{\uparrow}$ & $6 \uparrow$ & $9 \uparrow \longrightarrow$ & $12 \uparrow \longrightarrow$ & $12^{\uparrow} \longrightarrow$ & 1 & 3 & 6 & 9 & 12 & 12 & 12 \\
\hline 2 & $1 \uparrow$ & $2 \uparrow$ & $3 \stackrel{\uparrow}{\longrightarrow}$ & $3 \uparrow \longrightarrow$ & $3^{\uparrow}$ & {$[0]_{\downarrow} \searrow$} & 1 & 2 & 3 & 3 & 3 & 0 & 0 \\
\hline 1 & $1 \uparrow$ & $1 \uparrow$ & $\mathbf{1}_{\searrow}^{\uparrow} \rightarrow$ & {$[0]$} & {$[0]^{\prime}$} & 3 & 1 & 1 & 1 & 0 & 0 & -3 & 0 \\
\hline 0 & $\mathbf{1}_{\searrow}^{\uparrow}$ & {$[0]_{\downarrow}$} & {$[0]_{\downarrow} \searrow$} & $\mathbf{1}_{\downarrow \searrow}$ & \begin{tabular}{|l|}
$0_{\downarrow}$ \\
\end{tabular} & $3_{\downarrow} \searrow$ & 1 & 0 & 0 & -1 & 0 & -3 & 3 \\
\hline-1 & & $\mathbf{1}_{\searrow}$ & $\overline{0_{\downarrow}}$ & $1_{\downarrow \searrow}$ & $1_{\downarrow}$ & $3 \downarrow \searrow$ & & - & 0 & -1 & 1 & -3 & 3 \\
\hline-2 & & & $1 \searrow$ & $1_{\downarrow}$ & $2 \downarrow \searrow$ & $4_{\downarrow}$ & & & 1 & -1 & 2 & -4 & 9 \\
\hline-3 & & & & 2 & $3 \downarrow \searrow$ & $6_{\downarrow} \searrow$ & & & & -2 & 3 & -6 & 13 \\
\hline-4 & & & & & $5 \searrow$ & $9_{\downarrow \searrow}$ & & & $m)$ & & 5 & -9 & 19 \\
\hline-5 & & & & & & $14 \searrow$ & & & & & & -14 & 28 \\
\hline & 0 & 1 & 2 & 3 & 4 & 5 & $\overline{0}$ & 1 & 2 & 3 & 4 & 5 & $n$ \\
\hline
\end{tabular}

Table 5: Unifying the recursion

$(2 m+2, m)$ the path can take the steps $\searrow$ and $\downarrow$. The conjectured $n$-th Catalan number $C_{n}$ has moved down the diagonal to the position $(n+1,-n-1)$.

In the second step we change the sign of the entries in odd numbered columns, but only below the crack; call the new values $d(n, m)$. We have $d(n, m)=(-1)^{n} t^{\prime}(n, m)$ if $n \geq 2 m+2$, and $d(n, m)=t^{\prime}(n, m)$ if $n \leq 2 m+1$. We claim that

$$
d(n, m)=d(n, m-1)+d(n-1, m)
$$

for all $m \geq-n$. This trivially holds for $n \leq 2 m+2$, because it was already true for $t(n, m)$, and we filled the crack with the right values. If $n \geq 2 m+2$ and $n \geq-m$ then

$$
\begin{aligned}
d(n, m) & =(-1)^{n} t^{\prime}(n, m)=(-1)^{n}\left(t^{\prime}(n, m+1)+t^{\prime}(n-1, m+1)\right) \\
& =d(n, m+1)-d(n-1, m+1)
\end{aligned}
$$

confirming (1). Our conjecture about the Catalan numbers in Table $2, t(n,-n)=C_{n}$, is equivalent to the conjecture

$$
d(n+1,-n-1)=(-1)^{n+1} C_{n}
$$

for all $n \geq 0$. We prove this statement in two different approaches. The first proof, in Section 3 , shows that $d(n+1,-n-1)=(-1)^{n+1} C_{n}$ are the unique solutions of a system of equations associated to our problem. We solve the system in two ways, with and without the help of algebraic computer packages. The second proof interprets the numbers $d(n, m)$ above the crack as the number of lattice paths from the origin to $(n, m)$ strictly above the line $y=(x-1) / 2$. These numbers are known; their polynomial expansion shows that (2) holds.

Because of the simple recursion and initial values, there must be many other proofs of (2). However, the challenge is finding a combinatorial argument for the appearance of the nonalternating Catalan numbers $t(n,-n)=C_{n}$ in the original problem. 


\section{The Hypergeometric Function Approach}

The recursion (1) is a backwards difference recursion, $d(n, m)-d(n, m-1)=d(n-1, m)$, with initial values $d(0,0)=1$

$$
\begin{aligned}
d(n,(n-1) / 2) & =0 \text { for all odd } n>0 \\
d\left(n, \frac{n}{2}-1\right) & =0 \text { for all even } n>1 .
\end{aligned}
$$

Because we can extend $d(0, m)$ to the constant 1 for all $m \in \mathbb{Z}$, we can extend $d(n, m)$ to a polynomial $d_{n}(x)$ of degree $n$ such that $d_{n}(m)=d(n, m)$ for all $m \geq-n$.

$$
\begin{array}{r||cccccccc}
m & 1 & 3 & 6 & 9 & 12 & 12 & 12 & 0 \\
2 & 1 & 2 & 3 & 3 & 3 & 0 & 0 & -12 \\
1 & 1 & 1 & 1 & 0 & 0 & -3 & 0 & -12 \\
0 & 1 & 0 & 0 & -1 & 0 & -3 & 3 & -12 \\
-1 & 1 & -\mathbf{1} & 0 & -1 & 1 & -3 & 6 & -15 \\
-2 & 1 & -2 & \mathbf{1} & -1 & 2 & -4 & 9 & -21 \\
-3 & 1 & -3 & 3 & -\mathbf{2} & 3 & -6 & 13 & -30 \\
-4 & 1 & -4 & 6 & -5 & \mathbf{5} & -9 & 19 & -43 \\
-5 & 1 & -5 & 10 & -11 & 10 & -\mathbf{1 4} & 28 & -62 \\
\hline \hline & 0 & 1 & 2 & 3 & 4 & 5 & 6 & n
\end{array}
$$

Table 6: The polynomial extension $d_{n}(m)$ of $d(n, m)$

Any polynomial sequence $\left(s_{n}(x)\right)_{n>0}$ that satisfies the backwards difference equation $s_{n}(x)-s_{n}(x-1)=s_{n-1}(x)$ can be expanded as

$$
s_{n}(x)=\sum_{i=0}^{n} s_{i}(u i+v) \frac{x-u n-v}{x-u i-v}\left(\begin{array}{c}
n-i-1+x-u i-v \\
n-i
\end{array}\right)
$$

for any choice of the scalars $u$ and $v$ [7, Corollary 2.3]. Apply this expansion to the case $u=-1$ and $v=0$ to see that

$$
d_{n}(x)=\sum_{i=0}^{n} d_{i}(-i)\left(\begin{array}{c}
n+x \\
n-i
\end{array}\right)
$$

The initial values (3) imply that for all positive integers $n$

$$
0=\sum_{i=0}^{2 n-1} d_{i}(-i)\left(\begin{array}{c}
3 n-2 \\
2 n-1-i
\end{array}\right) \text { and } 0=\sum_{i=0}^{2 n} d_{i}(-i)\left(\begin{array}{c}
3 n-1 \\
2 n-i
\end{array}\right)
$$

This is a system of equations for the unknown coefficients $d_{i}(-i)$. The following proposition shows that $d_{i}(-i)=(-1)^{i} C_{i-1}$ are the unique solutions to the system if $d_{0}(0)=1$. This proves statement (2). Expansion (5) will then imply that

$$
d_{n}(x)=\left(\begin{array}{c}
x+n \\
n
\end{array}\right)+\sum_{j=1}^{n}(-1)^{j} C_{j-1}\left(\begin{array}{l}
x+n \\
n-j
\end{array}\right)
$$


hence we obtain the generating function

$$
\sum_{n \geq 0} d_{n}(x) t^{n}=\frac{3 \sqrt{1-t}-\sqrt{1+3 t}}{2(1-t)^{x+3 / 2}} .
$$

Proposition 1 For positive integers $n$ holds

$$
\begin{aligned}
& 0=\left(\begin{array}{c}
3 n-2 \\
2 n-1
\end{array}\right)+\sum_{i=1}^{2 n-1} \frac{(-1)^{i}}{i}\left(\begin{array}{c}
2 i-2 \\
i-1
\end{array}\right)\left(\begin{array}{c}
3 n-2 \\
2 n-1-i
\end{array}\right) \text { and } \\
& 0=\left(\begin{array}{c}
3 n-1 \\
2 n
\end{array}\right)+\sum_{i=1}^{2 n} \frac{(-1)^{i}}{i}\left(\begin{array}{c}
2 i-2 \\
i-1
\end{array}\right)\left(\begin{array}{c}
3 n-1 \\
2 n-i
\end{array}\right) .
\end{aligned}
$$

Equivalently,

$$
{ }_{2} F_{1}[-2 n+1,-1 / 2 ; n ; 4]={ }_{2} F_{1}[-2 n,-1 / 2 ; n ; 4]=3 .
$$

Recall that ${ }_{2} F_{1}[a, b ; c ; z]=1+\sum_{i \geq 1} \frac{a(a+1) \cdots(a+i-1) \cdot b(b+1) \cdots(b+i-1)}{i ! c(c+1) \cdots(c+i-1)} z^{i}$. See Subsections 3.1 and 3.2 for two different proofs of the proposition.

\subsection{Proof by Zeilberger's algorithm}

Let $f(n)={ }_{2} F_{1}[-2 n,-1 / 2 ; n ; 4]$ and $g(n)={ }_{2} F_{1}[-2 n+1,-1 / 2 ; n ; 4]$. Zeilberger's algorithm as implemented in Maple VII shows that

$$
\begin{aligned}
& f(n)=\frac{2\left(9 n^{2}-18 n+10\right) f(n-1)-9(n-1)(n-2) f(n-2)}{(3 n-1)(3 n-2)} \text { and } \\
& g(n)=\frac{2\left(9 n^{2}-27 n+22\right) g(n-1)-9(n-2)^{2} g(n-2)}{(3 n-2)(3 n-4)} .
\end{aligned}
$$

Both recursions are solved by constant functions. It is easy to check that $f(1)=g(1)=3$, hence $f(n)=g(n)=3$ for all positive integers $n$. This proves Proposition 1 in the form of equation (11).

\subsection{Proof by Gould's special hypergeometric function}

H.W. Gould [2] investigated the hypergeometric function ${ }_{2} F_{1}[-n, 1 / 2 ; m ; 4 z]$ for integers $m$ and positive integers $n$. Gould found integral representations and recursions involving ${ }_{2} F_{1}[-2 n, 1 / 2 ; m ; 4]$ and $F[-2 n+1,1 / 2 ; m ; 4]$. Important for this paper are his evaluations of the special cases $[3,(7.33)-(7.35),(7.40)-(7.42)]$

$$
\begin{array}{rrr}
{ }_{2} F_{1}[-2 n, 1 / 2 ; n ; 4]=3, & { }_{2} F_{1}[-2 n+1,1 / 2 ; n+1 ; 4]=0, \\
{ }_{2} F_{1}[-2 n+1,1 / 2 ; n ; 4]=-1 & { }_{2} F_{1}[-2 n, 1 / 2 ; n+2 ; 4] & =\frac{n+1}{2 n+1}, \\
{ }_{2} F_{1}[-2 n, 1 / 2 ; n+1 ; 4]=1, & { }_{2} F_{1}[-2 n+1,1 / 2 ; n+2 ; 4] & =\frac{n+1}{2(2 n+1)} .
\end{array}
$$

We also need the following technical lemma. 
Lemma 2 Let $n$ and $m$ be integers, $n>0$ and $m \neq 0$. Then

$$
{ }_{2} F_{1}[-n,-1 / 2 ; m ; 4 z]={ }_{2} F_{1}[-n, 1 / 2 ; m ; 4 z]+\frac{4 n z}{m}{ }_{2} F_{1}[-n+1,1 / 2 ; m+1 ; 4 z] \text {. }
$$

With the help of this Lemma and Gould's special values (12), (13) we prove Proposition 1 in the form of the identities (11),

$$
\begin{aligned}
{ }_{2} F_{1}[-2 n,-1 / 2 ; n ; 4] & ={ }_{2} F_{1}[-2 n, 1 / 2 ; n ; 4]+\frac{8 n}{n}{ }_{2} F_{1}[-2 n+1,1 / 2 ; n+1 ; 4]=3+0 \\
{ }_{2} F_{1}[-2 n+1,-1 / 2 ; n ; 4] & ={ }_{2} F_{1}[-2 n+1,1 / 2 ; n ; 4]+\frac{4(2 n-1)}{n}{ }_{2} F_{1}[-2 n+2,1 / 2 ; n+1 ; 4] \\
& =-1+4 .
\end{aligned}
$$

There are many companions to the the pair of identities in Proposition 1 . We prove one example.

Corollary 3 For positive integers $n$ holds

$$
\left(\begin{array}{c}
3 n \\
2 n+1
\end{array}\right)=\frac{1}{2} \sum_{i=0}^{2 n-1}(-1)^{i} C_{i}\left(\begin{array}{c}
3 n \\
2 n-1-i
\end{array}\right), \frac{n+1}{n}\left(\begin{array}{c}
3 n \\
2 n+1
\end{array}\right)=\sum_{i=0}^{2 n}(-1)^{i} C_{i}\left(\begin{array}{c}
3 n \\
2 n-i
\end{array}\right) .
$$

Proof. The special values (14) show that

$$
1+\frac{4 n}{2 n+1}=1+\frac{8 n}{n+1} \frac{n+1}{2(2 n+1)}={ }_{2} F_{1}[-2 n, 1 / 2 ; n+1 ; 4]+\frac{8 n}{n+1}{ }_{2} F_{1}[-2 n+1,1 / 2 ; n+2 ; 4] \text {. }
$$
By Lemma 2 thus

$$
1+\frac{4 n}{2 n+1}={ }_{2} F_{1}[-2 n,-1 / 2 ; n+1 ; 4]=\sum_{j=0}^{2 n} \frac{(-1 / 2)_{j} 2^{2 j}}{j !} \frac{(-2 n)_{j}}{(n+1)_{j}}=1-2 \sum_{j=1}^{2 n} \frac{(2 j-2) !(-2 n)_{j}}{j !(j-1) !(n+1)_{j}}
$$

$$
0=2\left(\begin{array}{c}
3 n \\
2 n+1
\end{array}\right)-\sum_{j=0}^{2 n-1} \frac{(-1)^{j}}{j+1}\left(\begin{array}{c}
2 j \\
j
\end{array}\right)\left(\begin{array}{c}
3 n \\
2 n-1-j
\end{array}\right) .
$$

This identity together with $\left(\begin{array}{l}3 n-2 \\ 2 n-1\end{array}\right)=\sum_{i=0}^{2 n-2}(-1)^{i} C_{i}\left(\begin{array}{c}3 n-2 \\ 2 n-2-i\end{array}\right)$ (see Proposition 1) imply that

$$
\frac{n+1}{n}\left(\begin{array}{c}
3 n \\
2 n+1
\end{array}\right)=\left(\begin{array}{c}
3 n+1 \\
2 n+1
\end{array}\right)-2\left(\begin{array}{c}
3 n \\
2 n+1
\end{array}\right)=\sum_{i=0}^{2 n} \frac{(-1)^{i}}{i+1}\left(\begin{array}{c}
2 i \\
i
\end{array}\right)\left(\begin{array}{c}
3 n \\
2 n-i
\end{array}\right) \text {. }
$$

\section{The Lattice Path Approach}

Let $D_{y=(x-c) / g}^{(n, m)}$ be the number of $\rightarrow, \uparrow$-paths strictly above $y=(x-c) / g$ from the origin to $(n, m)$, where $c$ is any non-negative integer. The untangled detours (Table 5) show that $d(n, m)=D_{y=(x-1) / 2}^{(n, m)}$. Because classical lattice path enumeration usually considers paths above lines of integer slope, we use two bijections on $\rightarrow$, $\uparrow$-paths to find 
$D_{y=(x-c) / g}^{(n, m)}$. Going backwards, from the end to $(0,0)$, through any path strictly above $y=(x-c) / g$ to $(n, d+(n-c) / g)$ yields a unique path strictly below $y=d+x / g$, again to $(n, d+(n-c) / g$ ), and vice versa (note that $d+(n-c) / g$ must be an integer). The second bijection reflects this new path at the diagonal $y=x$ onto a path strictly above $y=g(x-d)$, ending at $(d+(n-c) / g, n)$.

\begin{tabular}{|c|c|c|c|c|c|c|c|c|c|c|c|c|c|c|c|}
\hline \multicolumn{8}{|c|}{$D_{y=(x-1) / 2}^{(n, m)}(\#$ paths to $(6,5))$} & \multicolumn{8}{|c|}{$D_{y=2 x-5}^{(n, m)}(\#$ paths to $(5,6))$} \\
\hline$m$ & & & & & & & & $m$ & 1 & 7 & 28 & 80 & 163 & 163 & \\
\hline 5 & 1 & 5 & 15 & 34 & 65 & 108 & 163 & 5 & 1 & 6 & 21 & 52 & 83 & 0 & \\
\hline 4 & 1 & 4 & 10 & 19 & 31 & 43 & 55 & 4 & 1 & 5 & 15 & 31 & 31 & & \\
\hline 3 & 1 & 3 & 6 & 9 & 12 & 12 & 12 & 3 & 1 & 4 & 10 & 16 & 0 & & \\
\hline 2 & 1 & 2 & 3 & 3 & 3 & 0 & 0 & 2 & 1 & 3 & 6 & 6 & & & \\
\hline 1 & 1 & 1 & 1 & 0 & 0 & & & 1 & 1 & 2 & 3 & 0 & & & \\
\hline \multirow[t]{2}{*}{0} & 1 & 0 & 0 & & & & & 0 & 1 & 1 & 1 & & & & \\
\hline & $\overline{0} 0$ & 1 & 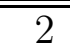 & 3 & 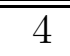 & 5 & $\bar{n}$ & & $\overline{00}$ & $\bar{~} 1$ & 2 & $\overline{3} 3$ & $\overline{4}$ & 5 & $n$ \\
\hline
\end{tabular}

Table 7: Two different lattice path problems arriving at the same number

The result is a path above a line with positive integer slope $g$, and we have established that $D_{y=(x-c) / g}^{(n, d+(n-c) / g)}=D_{y=g(x-d)}^{(d+(n-c) / g, n)}$. The number of paths above a line with positive integer slope is well known to be

$$
\begin{aligned}
D_{y=g(x-d)}^{(m, n)} & =\sum_{i=0}^{\lfloor d\rfloor}\left(\begin{array}{c}
i+g(i-d) \\
i
\end{array}\right) \frac{n-g(m-d)}{n-g(i-d)}\left(\begin{array}{c}
m-i-1+n-g(i-d) \\
m-i
\end{array}\right) \\
& =\left(\begin{array}{c}
m+n \\
m
\end{array}\right)-\sum_{i=\lfloor d\rfloor+1}^{m}\left(\begin{array}{c}
i+g(i-d) \\
i
\end{array}\right) \frac{n-g(m-d)}{n-g(i-d)}\left(\begin{array}{c}
m-i-1+n-g(i-d) \\
m-i
\end{array}\right)
\end{aligned}
$$

for $m \geq g(m-d)$ (for references see Koroljuk [4] or Mohanty [6]. The result also follows from (4), with $u=g, v=-g d$, and $s_{i}(u i+v)=\left(\begin{array}{c}i+g(i-d) \\ i\end{array}\right)$ for $i=0 \leq i \leq d$, and $s_{i}(u i+v)=0$ for $\left.i>d\right)$. In honor of Koroljuk's work we will also give a combinatorial proof of the formula for $D_{y=(x-c) / g}^{(n, m)}$ using his method at the end of this paper, in Proposition 4 .

We just saw that $D_{y=2(x-m+(n-1) / 2)}^{(m, n)}=D_{y=(x-1) / 2}^{(n, m)}=d(n, m)$, and therefore

$$
\begin{aligned}
d(n, m) & =\sum_{i=0}^{m-(n-1) / 2}\left(\begin{array}{c}
3 i-2 m+n-1 \\
i
\end{array}\right) \frac{1}{2(m-i)+1}\left(\begin{array}{c}
3(m-i) \\
m-i
\end{array}\right) \\
& =\left(\begin{array}{c}
m+n \\
m
\end{array}\right)-\sum_{i=0}^{(n-1) / 2} C_{i}^{\prime \prime \prime}\left(\begin{array}{c}
m-3 i-1+n \\
m-i
\end{array}\right) \\
& =\left(\begin{array}{c}
m+n \\
n
\end{array}\right)-(-1)^{n-1} \sum_{i=0}^{(n-1) / 2} C_{i}^{\prime \prime \prime}\left(\begin{array}{c}
i-m-1 \\
n-1-2 i
\end{array}\right)
\end{aligned}
$$


for $m \geq(n-1) / 2$ (remember that $C_{i}^{\prime \prime \prime}=\left(\begin{array}{c}3 i \\ i\end{array}\right) /(2 i+1)$, the Tri-Catalan numbers). Special values, like $2 m=n-1$, will confirm some of the identities in Corollary 3. The last of the three expansions of $d(n, m)$ in $(17)$ confirms that $d(n, m)$ is a polynomial in $m$ of degree $n$. Hence for $n \geq 1$ we obtain $d(n,-n)=$

$$
(-1)^{n} \sum_{i=0}^{(n-1) / 2}\left(\begin{array}{c}
n-1+i \\
3 i
\end{array}\right) \frac{1}{2 i+1}\left(\begin{array}{c}
3 i \\
i
\end{array}\right)=\frac{(-1)^{n}}{n} \sum_{i=0}^{(n-1) / 2}\left(\begin{array}{c}
n \\
2 i+1
\end{array}\right)\left(\begin{array}{c}
n+i-1 \\
i
\end{array}\right) .
$$

We can finish this lattice path proof of $d(n,-n)=(-1)^{n} C_{n-1}$ by showing that the latter sum equals $\left(\begin{array}{c}2 n-2 \\ n-1\end{array}\right)$ (see $\left.[3,(3.169)]\right)$, but it is also instructive to compare the generating functions. First notice that $\sum_{n=0}^{\infty}\left(\begin{array}{c}n+3 i \\ 3 i\end{array}\right) t^{n}=(1-t)^{-3 i-1}$ and

$$
\sum_{i=0}^{\infty} C_{i}^{\prime \prime \prime} t^{i}=\sum_{i=0}^{\infty} \frac{1}{2 i+1}\left(\begin{array}{c}
3 i \\
i
\end{array}\right) t^{i}={ }_{2} F_{1}\left[\frac{1}{3}, \frac{2}{3} ; \frac{3}{2} ; \frac{27}{4} t\right]=\frac{3 \sin \left(\frac{1}{3} \arcsin (\sqrt{27 t} / 2)\right)}{\sqrt{27 t} / 2}
$$

Thus

$$
\begin{aligned}
\sum_{n=1}^{\infty} t^{n-1}(-1)^{n} d(n,-n) & =\sum_{i=0}^{\infty} C_{i}^{\prime \prime \prime} \sum_{n=2 i+1}^{\infty} t^{n-1}\left(\begin{array}{c}
n-1+i \\
3 i
\end{array}\right)=\sum_{i=0}^{\infty} C_{i}^{\prime \prime \prime} t^{2 i}(1-t)^{-3 i-1} \\
& =\frac{1}{1-t}{ }_{2} F_{1}\left[\frac{1}{3}, \frac{2}{3} ; \frac{3}{2} ; U^{2}\right]=\frac{3 \sin \left(\frac{1}{3} \arcsin U\right)}{(1-t) U}
\end{aligned}
$$

where $U=\frac{3}{2} \sqrt{3 t^{2} /(1-t)^{3}}$. We can 'eliminate' the $1 / 3$ in front of the arcsin by finding a $\theta$ such that $\sin 3 \theta=U$. Define $\theta$ such that $\sin \theta=U(1-t)(1-\sqrt{1-4 t}) /(6 t)$. Applying the triple-angle formula $\sin 3 \theta=3 \sin \theta-4 \sin ^{3} \theta$ shows that indeed $\sin 3 \theta=U$. Hence

$$
\begin{aligned}
\sum_{n=1}^{\infty} t^{n-1}(-1)^{n} d(n,-n) & =\frac{3 \sin (\theta)}{(1-t) U}=\frac{3(1-t)}{(1-t) 6 t}(1-\sqrt{1-4 t})=\frac{1-\sqrt{1-4 t}}{2 t} \\
& =\frac{2}{1+\sqrt{1-4 t}}=\sum_{n \geq 0} C_{n} t^{n}
\end{aligned}
$$

the well-known generating function of the Catalan numbers (I thank the referee for shortening the derivation by means of the triple-angle formula). We will have another look at this generating function identity in Section 5.3.

\section{Some Remarks on Paths above a Fractional Slope}

There are more types of known number sequences occurring in the Catalan traffic scheme than just the Catalan numbers, and there are other lattice path problems restricted by a boundary line of slope $1 / 2$ where the alternating Catalan numbers play a role. A short discussion follows in the next two subsections. Finally, we finish with a glimpse at the enumeration of lattice paths above a line of fractional slope. 


\subsection{Other types of numbers generated by Catalan traffic}

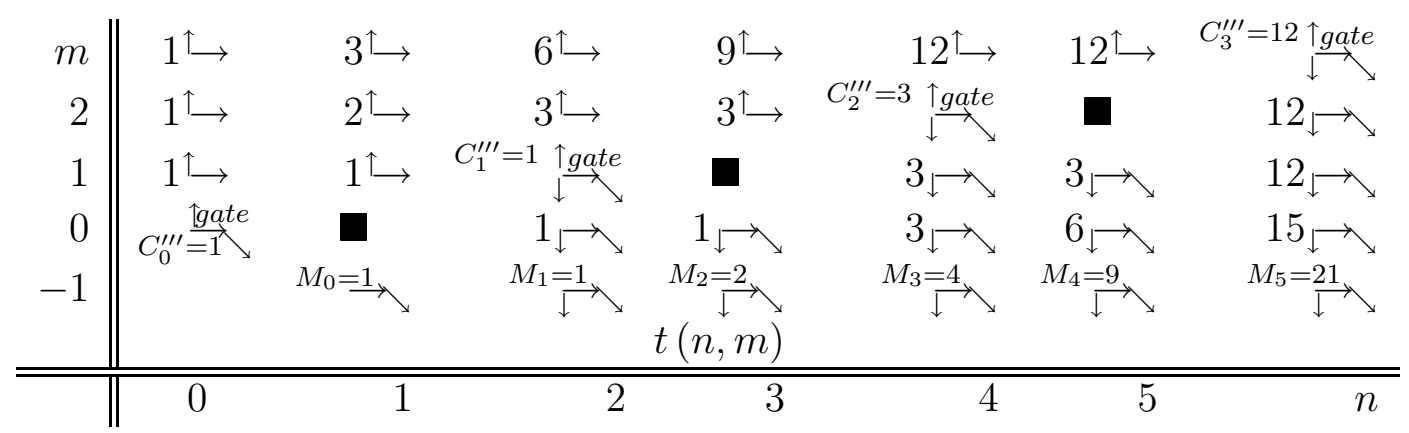

Table 8: Motzkin and Tri-Catalan numbers in the Catalan traffic table

Formulas (7) and (17) are simple expressions for the number $D_{y=(x-1) / 2}^{(n, m)}$ of $\rightarrow, \uparrow$-lattice paths from the origin to $(n, m)$ staying strictly above the boundary $y=(x-1) / 2$,

$$
\begin{aligned}
D_{y=(x-1) / 2}^{(n, m)} & =d(n, m)=\left(\begin{array}{c}
m+n \\
n
\end{array}\right)-\sum_{j=0}^{n-1}(-1)^{j} C_{j}\left(\begin{array}{c}
m+n \\
n-1-j
\end{array}\right) \\
& =\left(\begin{array}{c}
m+n \\
m
\end{array}\right)+(-1)^{n} \sum_{i=0}^{(n-1) / 2} C_{i}^{\prime \prime \prime}\left(\begin{array}{c}
i-m-1 \\
n-1-2 i
\end{array}\right)
\end{aligned}
$$

for all $n \leq 2 m+1$, and also for the polynomial extension to other values of $m$ (again, we use the abbreviation $\left.C_{i}^{\prime \prime \prime}=\left(\begin{array}{c}3 i \\ i\end{array}\right) /(2 i+1)\right)$. It is now easy to prove that the Motzkin numbers $M_{n}=\frac{1}{n+1} \sum_{k=0}^{n}\left(\begin{array}{c}k \\ n-k\end{array}\right)\left(\begin{array}{c}n+1 \\ k\end{array}\right)$ occur as $t(n-1,-1)$ in the Catalan traffic Table 2, and equivalently as $(-1)^{n} d(n+2,-2)$ in Table 6 . To check this, note that $\sum_{n \geq 0} d_{n}(-2) t^{n}=$ $\left(3(1-t)-\sqrt{1+2 t-3 t^{2}}\right) / 2$, a special case of the generating function $\sum_{n \geq 0} d_{n}(x) t^{n}=$ $(3 \sqrt{1-t}-\sqrt{1+3 t})(1-t)^{-x-3 / 2} / 2($ see $(8))$. Hence

$$
\sum_{n \geq 2}(-1)^{n-1} d_{n}(-2) t^{n-2}=t^{-2}(1-t-\sqrt{(1-3 t)(1+t)}) / 2,
$$

the generating function of the Motzkin numbers.

We can apply the expansion $d_{n}(x)=\sum_{i=0}^{n} d_{i}(u i+v) \frac{x-u n-v}{x-u i-v}\left(\begin{array}{c}n-i-1+x-u i-v \\ n-i\end{array}\right)$ (see (4)) to express the numbers $d(n, m)$ in terms of the Motzkin numbers,

$$
\begin{aligned}
d(n, m) & =\sum_{i=0}^{n} d_{i}(-2)\left(\begin{array}{c}
n-i+1+m \\
n-i
\end{array}\right) \\
& =\left(\begin{array}{c}
n+1+m \\
n
\end{array}\right)-2\left(\begin{array}{c}
n+m \\
n-1
\end{array}\right)+\sum_{i=0}^{n-2} d_{i+2}(-2)\left(\begin{array}{c}
n-i-1+m \\
n-2-i
\end{array}\right) \\
& =\frac{m-n+1}{m+1}\left(\begin{array}{c}
n+m \\
n
\end{array}\right)+\sum_{i=0}^{n-2}(-1)^{i} M_{i}\left(\begin{array}{c}
n-i-1+m \\
n-2-i
\end{array}\right) .
\end{aligned}
$$


The numbers at the gates in Table 2 , or $d(2 m, m)$ in Table 6 , are the Tri-Catalan numbers $C_{m}^{\prime \prime \prime}$; this follows from (19) and the first identity in Corollary 3 ,

$$
\begin{aligned}
d(2 m, m) & =\left(\begin{array}{c}
3 m \\
m
\end{array}\right)-\sum_{j=0}^{2 m-1}(-1)^{j} C_{j}\left(\begin{array}{c}
3 m \\
2 m-1-j
\end{array}\right)=\left(\begin{array}{c}
3 m \\
m
\end{array}\right)-2\left(\begin{array}{c}
3 m \\
2 m+1
\end{array}\right) \\
& =\frac{1}{2 m+1}\left(\begin{array}{c}
3 m \\
m
\end{array}\right)=C_{m}^{\prime \prime \prime} .
\end{aligned}
$$

In the following table we summarize the inverse relationships (in the sense of Riordan $[9$, Chpt. 2]) between the three types of numbers, Catalan, Motzkin, and Tri-Catalan, using (19), (20), and Corollary 3.

$$
\begin{aligned}
& C_{n}=(-1)^{n+1} d_{n+1}(-n-1)=\sum_{i=0}^{n / 2} C_{i}^{\prime \prime \prime}\left(\begin{array}{c}
i+n \\
n-2 i
\end{array}\right)=\sum_{i=0}^{n-1} M_{i}\left(\begin{array}{c}
n-1 \\
n-1-i
\end{array}\right) \\
& M_{n}=(-1)^{n} d(n+2,-2)=\sum_{i=0}^{(n+1) / 2} C_{i}^{\prime \prime \prime}\left(\begin{array}{c}
i+1 \\
n+1-2 i
\end{array}\right)=\sum_{i=0}^{n+1}(-1)^{n+1-i} C_{i}\left(\begin{array}{c}
n \\
n+1-i
\end{array}\right) \\
& C_{n}^{\prime \prime \prime}=d(2 n, n)=\frac{1-n}{n+1}\left(\begin{array}{c}
3 n \\
n
\end{array}\right)+\sum_{i=0}^{2 n-2}(-1)^{i} M_{i}\left(\begin{array}{c}
3 n-i-1 \\
n+1
\end{array}\right)=\sum_{i=0}^{2 n-1} \frac{(-1)^{i}}{2 n} C_{i}\left(\begin{array}{c}
3 n \\
2 n-1-i
\end{array}\right) .
\end{aligned}
$$

Because of the boundary $y=(x-1) / 2$ we have the initial values $d(2 m+1, m)=$ $d(2 m, m-1)=0$ for all $m>0$ in $(3)$, implying that $d(2 m, m)=d(2 m-1, m)=$ $d(2 m-2, m)$. Each of the three equal terms we can expand with formula (19), as we did for $d(2 m, m)$ above. Expanding the difference $d(2 m, m)-d(2 m-1, m)=0$ shows that

$$
\left(\begin{array}{c}
3 m-1 \\
m-1
\end{array}\right)=\sum_{j=0}^{2 m-1}(-1)^{j} C_{j}\left(\begin{array}{c}
3 m-1 \\
2 m-1-j
\end{array}\right)
$$

recovering the second equation in Proposition 1. The first follows analogously.

In Proposition 4 we will apply a standard combinatorial argument to prove that $d(2 m, m)=C_{m}^{\prime \prime \prime}$, without the help of Catalan numbers or any polynomial extensions. However, I did not find a direct combinatorial proof showing that the Catalan numbers must occur in the traffic scheme; all the arguments above are based on polynomial extensions of $D_{y=(x-1) / 2}^{(n, m)}$ to values that can not be interpreted as a number of lattice paths.

\subsection{Other lattice path problems generating alternating Catalan numbers}

There are closely related lattice path problems where the alternating Catalan numbers play a similar role. A trivial variation is the number $D_{y=c+(x-1) / 2}^{(n, m)}$ of all $\rightarrow$, $\uparrow$-paths from $(0,0)$ to $(n, m)$ staying strictly above $y=c+(x-1) / 2$, for some positive integer $c$. In 


\begin{tabular}{|c|c|c|c|c|c|c|c|c|c|c|c|c|c|c|}
\hline \multicolumn{8}{|c|}{$d_{n}(m)$} & \multicolumn{7}{|c|}{$f_{n}(m)$} \\
\hline$m$ & 1 & 4 & 10 & 19 & 31 & 43 & 55 & $\overline{1}$ & 4 & 9 & 16 & 23 & 30 & 30 \\
\hline 3 & 1 & 3 & 6 & 9 & 12 & 12 & 12 & 1 & 3 & 5 & 7 & 7 & 7 & 0 \\
\hline 2 & 1 & 2 & 3 & 3 & 3 & 0 & 0 & 1 & 2 & 2 & 2 & 0 & 0 & -7 \\
\hline 1 & 1 & 1 & 1 & 0 & 0 & -3 & 0 & 1 & 1 & 0 & 0 & -2 & 0 & -7 \\
\hline 0 & 1 & 0 & 0 & -1 & 0 & -3 & 3 & 1 & 0 & -1 & 0 & -2 & 2 & -7 \\
\hline-1 & 1 & -1 & 0 & -1 & 1 & -3 & 6 & 1 & -1 & -1 & 1 & -2 & 4 & -9 \\
\hline-2 & 1 & -2 & 1 & -1 & 2 & -4 & 9 & 1 & -2 & 0 & 2 & -3 & 6 & -13 \\
\hline-3 & 1 & -3 & 3 & -2 & 3 & -6 & 13 & 1 & -3 & 2 & 2 & -5 & 9 & -19 \\
\hline-4 & 1 & -4 & 6 & -5 & 5 & -9 & 19 & 1 & -4 & 5 & 0 & -7 & 14 & -28 \\
\hline-5 & 1 & -5 & 10 & -11 & 10 & -14 & 28 & 1 & -5 & 9 & -5 & -7 & 21 & -42 \\
\hline & $\overline{0}$ & 1 & 2 & $\overline{3}$ & 4 & 5 & $n$ & $\overline{00}$ & 1 & 2 & 3 & $\overline{4}$ & 5 & $n$ \\
\hline
\end{tabular}

Table 9: The polynomial extensions $d_{n}(m)$ of $d(n, m)$, and $f_{n}(m)$ of $f(n, m)$

this case all paths begin with upwards steps, and therefore $D_{y=c+(x-1) / 2}^{(n, m)}=D_{y=(x-1) / 2}^{(n, m-c)}=$ $d(n, m-c)$. Hence $D_{y=c+(x-1) / 2}^{(i, c-i)}=d(i,-i)=(-1)^{i-1} C_{i-1}$.

In Table 9 the numbers $f(n, m):=D_{y=x / 2}^{(n, m)}$ of all $\rightarrow$, $\uparrow$-paths from $(0,0)$ to $(n, m)$ staying strictly above $y=x / 2$ (except at the origin) look very different from $d(n, m)$ - except for the alternating Catalan numbers! We will show below that $f(i+1,-i)=$ $(-1)^{i} C_{i}$ for positive $i$ (note that $f(1,0)=0$ and $f(0,1)=1$ ). The backwards difference recursion for those paths has initial values $f(2 m, m)=f(2 m+1, m)=0$, except for $f(0,0)=1$. We apply the expansion $f_{n}(x)=\sum_{i=0}^{n} f_{i}(u i+v) \frac{x-u n-v}{x-u i-v}\left(\begin{array}{c}n-i-1+x-u i-v \\ n-i\end{array}\right)$ (see (4)) to the polynomial extension $f_{n}(x)$ of $f(n, m)$, with $u=-1$ and $v=1$

$$
f_{n}(x)=\sum_{i=0}^{n} f_{i}(1-i)\left(\begin{array}{c}
n-1+x \\
n-i
\end{array}\right)=\left(\begin{array}{c}
n-1+x \\
n
\end{array}\right)+\sum_{i=2}^{n} f_{i}(1-i)\left(\begin{array}{c}
n-1+x \\
n-i
\end{array}\right) .
$$

Substituting the initial values $f(2 m, m)=\delta_{0, m}$ and $f(2 m+1, m)=0$ shows that

$$
\left(\begin{array}{c}
3 m-1 \\
2 m
\end{array}\right)=-\sum_{i=2}^{2 m} f_{i}(1-i)\left(\begin{array}{c}
3 m-1 \\
2 m-i
\end{array}\right) \text { and }\left(\begin{array}{c}
3 m \\
2 m+1
\end{array}\right)=-\sum_{i=2}^{2 m+1} f_{i}(1-i)\left(\begin{array}{c}
3 m \\
2 m+1-i
\end{array}\right)
$$

Therefore we can determine $f_{i}(1-i)$ as the unique solutions of the system of equations

$$
\begin{aligned}
\left(\begin{array}{c}
3 m-1 \\
2 m
\end{array}\right) & =\left(\begin{array}{c}
3 m-1 \\
2 m-1
\end{array}\right)-\left(\begin{array}{c}
3 m-1 \\
2 m
\end{array}\right)=\left(\begin{array}{c}
3 m-1 \\
2 m-1
\end{array}\right)+\sum_{i=1}^{2 m-1} f_{i+1}(-i)\left(\begin{array}{c}
3 m-1 \\
2 m-1-i
\end{array}\right), \\
\frac{m+1}{m}\left(\begin{array}{c}
3 m \\
2 m+1
\end{array}\right) & =\left(\begin{array}{c}
3 m \\
2 m
\end{array}\right)-\left(\begin{array}{c}
3 m \\
2 m+1
\end{array}\right)=\left(\begin{array}{c}
3 m \\
2 m
\end{array}\right)+\sum_{i=1}^{2 m} f_{i+1}(-i)\left(\begin{array}{c}
3 m \\
2 m-i
\end{array}\right) .
\end{aligned}
$$

From equation (10) in Proposition 1 and the second equation in Corollary 3 follows 
$f_{i+1}(-i)=(-1)^{i} C_{i}$ for positive $i$. Hence

$$
\begin{aligned}
f_{n}(x) & =\left(\begin{array}{c}
n-1+x \\
n
\end{array}\right)+\sum_{i=1}^{n-1}\left(\begin{array}{c}
n-1+x \\
n-1-i
\end{array}\right) \text { and } \\
\sum_{n=0}^{\infty} f_{n}(x) t^{n} & =\frac{1}{2}(1-t)^{-x-1}(1-3 t+\sqrt{(3 t+1)(1-t)}) .
\end{aligned}
$$

The special cases

$$
f(2 m-3, m)=f(2 m-2, m)=f(2 m-1, m)=\left(\begin{array}{c}
3 m-2 \\
2 m-1
\end{array}\right)+\sum_{i=1}^{2 m-2}(-1)^{i} C_{i}\left(\begin{array}{c}
3 m-2 \\
2 m-2-i
\end{array}\right)
$$

can be summed, because by $(9)$

$$
\sum_{i=1}^{2 m-2}(-1)^{i} C_{i}\left(\begin{array}{c}
3 m-2 \\
2 m-2-i
\end{array}\right)=\left(\begin{array}{c}
3 m-2 \\
2 m-1
\end{array}\right)-\left(\begin{array}{c}
3 m-2 \\
2 m-2
\end{array}\right)
$$

thus $f(2 m-3, m)=f(2 m-2, m)=$

$$
f(2 m-1, m)=2\left(\begin{array}{l}
3 m-2 \\
2 m-1
\end{array}\right)-\left(\begin{array}{l}
3 m-2 \\
2 m-2
\end{array}\right)=\frac{1}{2 m-1}\left(\begin{array}{l}
3 m-2 \\
2 m-2
\end{array}\right)
$$

The numbers $\frac{1}{2 m-1}\left(\begin{array}{c}3 m-2 \\ 2 m-2\end{array}\right)=\frac{2}{3}\left(\begin{array}{c}3 m \\ 2 m\end{array}\right) /(3 m-1), m \geq 1$, are sequence A006013 in the OnLine Encyclopedia of Integer Sequences. They occur in the enumeration of noncrossing trees on a circle [8].

Again, a trivial variation is the number $D_{y=c+x / 2}^{(n, m)}=f(n, m-c)$ of all $\rightarrow$, $\uparrow$-paths from $(0,0)$ to $(n, m)$ staying strictly above $y=c+x / 2$, for some positive integer $c$.

\subsection{Slopes that are half-integers}

Applying his probabilistic method, Ira Gessel [1] found generating functions for enumerating paths below half-integer slopes. Let $\lambda$ be a nonnegative, $s$ a positive integer, and denote by $g_{m}$ the number of $\rightarrow$, $\uparrow$-paths strictly below $y=\frac{2 \lambda+1}{2} x+s-1 / 2$ to $\left(2 m, s+\left(\lambda+\frac{1}{2}\right) 2 m\right)$, and by $h_{m}$ the number of paths strictly below $y=\frac{2 \lambda+1}{2} x+s$ to $\left(2 m+1, s-\frac{1}{2}+\left(\lambda+\frac{1}{2}\right)(2 m+1)\right)$. Let $q(t)$ be the power series defined by $t=$ $(1-q(t)) q(t)^{\lambda+1 / 2}$. Gessel showed by Lagrange inversion that

$$
q(t)^{-a}=\sum_{m=0}^{\infty} \frac{a}{(\lambda+3 / 2) m+a}\left(\begin{array}{c}
(\lambda+3 / 2) m+a \\
m
\end{array}\right) t^{m} .
$$

For example, let $a=1, \lambda=3 / 2$, and we will obtain the generating function of the Tri-Catalan numbers, if we only solve $t=(1-q(t)) q(t)^{2}$ for $q(t)$. This is not a simple expression; however, we observed in Section 4 that the substitution $t=\tau^{2}(1-\tau)^{-3}$ enormously simplifies the formal power series, giving us $q(\tau)=\frac{1}{2}(1-\sqrt{1-4 \tau})(1-\tau)$.

Gessel's probabilistic method shows that

$$
\sum_{m=0}^{\infty} g_{m} t^{2 m}=\frac{q(t)^{-s+1 / 2}+q(-t)^{-s+1 / 2}}{q(t)^{1 / 2}+q(-t)^{1 / 2}} \text { and } \sum_{m=0}^{\infty} h_{m} t^{2 m+1}=\frac{q(t)^{-s}+q(-t)^{-s}}{q(t)^{-1 / 2}+q(-t)^{-1 / 2}}
$$


As an example, Gessel evaluated the case $s=1$, and found

$$
\begin{aligned}
h_{m} & =\frac{1}{(2 \lambda+3) m+\lambda+2}\left(\begin{array}{c}
(2 \lambda+3) m+\lambda+2 \\
2 m+1
\end{array}\right) \\
& =\frac{1}{(\lambda+3 / 2)(2 m+1)+1 / 2}\left(\begin{array}{c}
(\lambda+3 / 2)(2 m+1)+1 / 2 \\
2 m+1
\end{array}\right),
\end{aligned}
$$

the number of paths strictly below $y=\frac{2 \lambda+1}{2} x+1$ to $\left(2 m+1, \frac{1}{2}+\left(\lambda+\frac{1}{2}\right)(2 m+1)\right)$.

Traversing backwards through a path, from the end to the origin, shows that every path form $(0,0)$ to $\left(2 m+1, s-\frac{1}{2}+\frac{2 \lambda+1}{2}(2 m+1)\right)$ strictly below $y=s-\frac{1}{2}+\frac{2 \lambda+1}{2} x$ (except at the end point), corresponds to a path from $(0,0)$ to $\left(2 m+1, s-\frac{1}{2}+\frac{2 \lambda+1}{2}(2 m+1)\right)$, strictly above $y=\frac{2 \lambda+1}{2} x$. If $\lambda=0$ and $s=1$, this becomes a path from $(0,0)$ to $(2 m+1, m+1)$, strictly above $y=x / 2$, and

$$
h_{m}=\frac{1}{3 m+2}\left(\begin{array}{l}
3 m+2 \\
2 m+1
\end{array}\right)=\frac{1}{3 m+2}\left(\begin{array}{c}
3 m+2 \\
2 m+1
\end{array}\right)=\frac{1}{2 m+1}\left(\begin{array}{c}
3 m+1 \\
2 m
\end{array}\right)=f(2 m+1, m+1)
$$

according to (22) as well as (21) in the preceding subsection.

\subsection{Slopes that are unit fractions}

Gessel's approach works well for half-integer slopes. For unit fractions, slopes of the form $1 / g$, we can find the number of paths from the bijections we discussed at the start of Section 4, showing that $D_{y=(x-d) / g}^{(n, m)}=D_{y=g(x-m+(n-d) / g)}^{(m, n)}$. The following elementary approach mirrors Koroljuk's method for positive integer slopes [4].

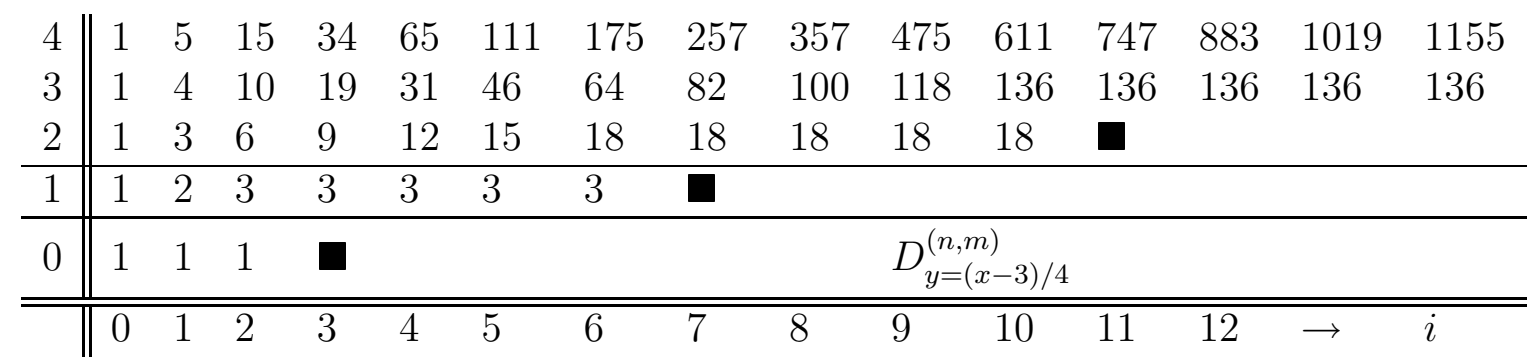

Table 10: $\{\rightarrow \uparrow\}$-paths strictly above $4 y+3=x$

Proposition 4 Suppose $g$ and $c$ are positive integers, and $\mathcal{D}_{y=(x-c) / g}^{(k)}$ is the set of $\rightarrow, \uparrow-$ paths from the origin to $(g k+c, k)$ staying strictly above the boundary $g y+c=x$, except at the end point $(g k+c, k)$. Then

$$
\left|\mathcal{D}_{y=(x-c) / g}^{(g k+c, k)}\right|=\frac{c}{(g+1) k+c}\left(\begin{array}{c}
(g+1) k+c \\
k
\end{array}\right),
$$


a generalized Catalan number. Furthermore,

$$
D_{y=(x-c) / g}^{(n, m)}=\left(\begin{array}{c}
n+m \\
n
\end{array}\right)-\sum_{i=0}^{(n-c) / g}\left(\begin{array}{c}
(g+1) i+c \\
i
\end{array}\right)\left(\begin{array}{c}
n+m-(g+1) i-c \\
m-i
\end{array}\right) \frac{c}{(g+1) i+c} .
$$

Proof. Define $D_{y=(x-c) / g}^{(k)}=\left|\mathcal{D}_{y=(x-c) / g}^{(g k+c, k)}\right|$. Any (unrestricted) path from $(0,0)$ to $(g k+c, k)$ can be decomposed into a head piece that reaches the line $g y=x-c$ for the first time at $(g j+c, j)$, say, and a tail from $(g j+c, j)$ to $(g k+c, k)$ without any restrictions. Hence

$$
\left(\begin{array}{c}
g k+c+k \\
k
\end{array}\right)=\sum_{i=0}^{k} D_{y=(x-c) / g}^{(i)}\left(\begin{array}{c}
g(k-i)+k-i \\
k-i
\end{array}\right) .
$$

Applying the expansion $s_{k}(g k+c)=\sum_{i=0}^{k} s_{i}(g i) \frac{c}{(g+1)(k-i)+c}\left(\begin{array}{c}(g+1)(k-i)+c \\ k-i\end{array}\right)$ to the polynomials $s_{n}(x)=\left(\begin{array}{c}g n+x \\ n\end{array}\right)($ see $(4))$ shows the well-known identity

$$
\left(\begin{array}{c}
(g+1) k+c \\
k
\end{array}\right)=\sum_{i=0}^{k}\left(\begin{array}{c}
g i+i \\
i
\end{array}\right) \frac{c}{(g+1)(k-i)+c}\left(\begin{array}{c}
(g+1)(k-i)+c \\
k-i
\end{array}\right) .
$$

Hence $D_{y=(x-c) / g}^{(i)}=\frac{c}{(g+1) k+c}\left(\begin{array}{c}(g+1) k+c \\ k\end{array}\right)$. We apply the idea of head and tail pieces again, but now to the path from $(0,0)$ to $(n, m)$ that reach at least one point on the line $g y+c=$ $x$. There are $\sum_{i=0}^{(n-c) / g} D_{y=(x-c) / g}^{(i)}\left(\begin{array}{c}n-g i-c+m-i \\ m-i\end{array}\right)$ such paths. This proves the formula for $D_{y=(x-c) / g}^{(n, m)}$.

The generalized Catalan numbers occur in the enumeration of $g+1$-ary trees and the $g+1$-tennis ball problem [5]. For $g=2$ and $c=1$ the Proposition verifies the Tri-Catalan numbers at the gates in Table 2 , because $\left|\mathcal{D}_{y=(x-c) / g}^{(g k+c, k)}\right|=D_{y=(x-c) / g}^{(g k+c-1, k)}$.

\section{References}

[1] Gessel, I. (1986). A probabilistic method for lattice path enumeration. J. Statist. Plann. Inference 14, 49-58.

[2] Gould, H.W. (1973). Improved evaluation of the finite hypergeometric series $F(-n, 1 / 2 ; j+1)$. Proc. West Virginia Acad. Sci. 45, 317-323.

[3] Gould, H.W. (1972). Combinatorial Identities. Morgantown, Va.

[4] Koroljuk, V.S. (1955) On the discrepancy of empiric distributions for the case of two independent samples. Izvestiya Akad. Nauk SSSR. Ser. Mat. 19, 81-96 (translated in $I M S \& A M S 4$ (1963) 105-122).

[5] Merlini, D. G., Sprugnoli, R., and Verri, M. C. (2002). The tennis ball problem. To appear in J. Comb. Th. A. 
[6] Mohanty, S.G. (1979). Lattice Path Counting and Applications. Academic Press, New York.

[7] Niederhausen, H. (1980). Linear recurrences under side conditions. European J. Combin. 1, 353-368.

[8] Noy, M. (1998). Enumeration of noncrossing trees on a circle, Discr. Math. 180, $301-313$.

[9] Riordan, J. (1968). Combinatorial Identities, Wiley, New York.

[10] Stanley, R.P. (1999). Enumerative Combinatorics, Vol. II, Cambridge University Press, Cambridge, UK. 